\title{
Bowel Perforation Following Continuation of Bevacizumab Post Nasal Septal Perforation-A Case Report
}

\author{
D. J. Nelmes, S. H. Gwynne \\ Singleton Oncology Department, Singleton Hospital, Swansea, UK \\ Email: NelmesD@cardiff.ac.uk
}

Received 17 February 2014; revised 15 March 2014; accepted 14 April 2014

Copyright (C) 2014 by authors and Scientific Research Publishing Inc.

This work is licensed under the Creative Commons Attribution International License (CC BY). http://creativecommons.org/licenses/by/4.0/

(c) (i) Open Access

\begin{abstract}
We report the case of a 63-year-old male with an inoperableT4N1 adenocarcinoma of colon, $K$-RAS mutant, who received first line chemotherapy with capecitabine and oxaliplatin. A CT scan following 4 cycles demonstrated progressive disease, and second line therapy with capecitabine, irinotecan and bevacizumab was commenced. CT scans at 3 and 6 months during this treatment regime demonstrated radiologically stable disease, and therefore the treatment was continued. The patient developed nasal septal perforation, a rare but recognised complication of bevacizumab therapy, which was managed conservatively. Here we highlight that no consensus exists on whether bevacizumab should be continued in this situation. After a detailed discussion about the risks and benefits, this patient continued on with the same therapeutic regime. However, eight weeks later, this patient then developed a localised tumour perforation, necessitating an emergency admission to his local hospital. We recommend caution in continuing bevacizumab in patients with colorectal cancer following a nasal septal perforation and advise a detailed discussion of risk with the patient, especially when the primary tumour remains in-situ.
\end{abstract}

\section{Keywords}

Colorectal Cancer, Bevacizumab, Avastin, Nasal, Septal, Perforation

\section{Case History}

We report the case of Mr L., a 63-year-old man, who presented with an abdominal mass and hydronephrosis, and was found to have a T4N1 mucinous, poorly differentiated adenocarcinoma of the colon, which was K-RAS mutant. He had an attempted resection in July 2012 but the tumour was inoperable so nephrostomies were fa- 
shioned. This patient commenced on $1^{\text {st }}$ line chemotherapy with Capecitabine $\left(1000 \mathrm{mg} / \mathrm{m}^{2}\right.$.days 1 - 14$)$ and Oxaliplatin $\left(130 \mathrm{mg} / \mathrm{m}^{2}\right.$.day 1$)$, each on a 21 day cycle. A CT scan following 4 cycles of chemotherapy demonstrated progression of disease, and he was changed to second line therapy with Capecitabine $\left(1000 \mathrm{mg} / \mathrm{m}^{2} \cdot\right.$ days 1 - 14), Irinotecan $\left(250 \mathrm{mg} / \mathrm{m}^{2}\right.$.day 1$)$ and Bevacizumab $\left(7.5 \mathrm{mg} / \mathrm{m}^{2}\right.$.day 1$)$, each on a 21 day cycle. CT scans at 3 and 6 months during this treatment demonstrated radiological stable disease. This patient then presented with a self-detected perforation of the nasal septum. He was reviewed by ENT who did not recommend any intervention but planned for careful observation and monitoring. The risks (deterioration of nasal perforation and possibility of gastro-intestinal perforation), and benefits (evidence of tumour control), were discussed with the patient, and it was decided to continue with the Bevacizumab. Eight weeks later, RL was admitted to his local hospital with severe abdominal pain, and found to have localised tumour perforation. He was managed conservatively, and to date is making a slow recovery. Bevacizumab has now been discontinued indefinitely.

\section{Discussion}

Bevacizumab, or Avastin ${ }^{\circledR}$, is a recombinant humanised monoclonal antibody that targets human Vascular Enothelial Growth Factor (VEGF). It exerts an anti-cancer activity by preventing tumour angiogenesis, and inhibiting immature blood vessel formation, thus reducing the ability of cancer to develop and maintain a blood supply [1]. It is currently licensed for use in metastatic colorectal cancer in combination with fluoropyrimidines, however, its use is not currently supported by NICE (NICE Technology Appraisal TA212, December 2010) [2].

There is a $1 \%$ - 9\% reported incidence of nasal septal perforation with the use of Avastin ${ }^{\circledR}$, which may be higher in breast cancer patients, compared with colorectal cancer patients, possibly due to concurrent use of taxanes [3]-[5]. Nasal septal perforation is largely thought of as a non-life threatening, self-limiting event. Patients may experience noisy nasal airflow and/or epistaxis. It may be an under-reported adverse event, as not all patients receiving bevacizumab therapy, who experience epistaxis, have assessment by ENT [4].

There is very little guidance in the literature with regard to the management of bevacizumab induced nasal septal perforation, specifically whether or not treatment with bevacizumab should be continued. Malliez (2010) concluded that for this patient group, assessment and monitoring by an ENT specialist is warranted, but whether to continue bevacizumab is unclear. They report a cohort of patients treated with bevacizumab who had favourable tumour responses, despite the development of nasalseptal perforation, and without objective worsening of the defect. There is no data on whether nasal septal perforation heralds an increased risk of other perforations, e.g. gastro-intestinal perforations [5].

In a meta-analysis, the incidence of gastro-intestinal (GI) perforation, in patients being treated with bevacizumab, was $0.9 \%$. This is a significantly increased risk compared to controls (RR 2.14, 95\% CI 1.19-3.85, $\mathrm{p}=$ 0.011). The risk is higher for patients with colorectal cancer, and could be related to dose, comparing 5 $\mathrm{mg} / \mathrm{kg} /$ week (RR 6.95 (95\% CI 0.36-133.97)) with $2.5 \mathrm{mg} / \mathrm{kg} /$ week (RR 2.86 (95\% CI 1.11-7.35)) [6]. A subsequent literature review by Hompes (2011) [7] recounts that from the First BEAT study, patients with unresected primary tumours treated with bevacizumab and GI perforation was seen in 8 of 223 (4\%): only 3 of which occurring at the primary tumour site [8]. In the BRiTE study, GI perforation rate for this group of patients was 3\%, versus almost $2 \%$ in patients that the primary tumour had been resected [9]. Multivariate analysis rated this as an independent risk factor for GI perforation, but event numbers were low. Whether these perforations occurred at the primary tumour site, was not specified. Poultsides, et al., (2009) reveal that GI perforation usually occurs within the first 3 months of commencing bevacizumab therapy, though later onset cases have been reported, and they can occur anywhere along the GI tract [10].

In hindsight, the development of a nasal septal perforation in a patient with a primary tumour in-situ, might have led to a decision to discontinue bevacizumab at an earlier stage, but the risks and benefits of continuation of treatment were discussed with the patient and in the absence of a clear contraindication, the treatment was continued. We recommend caution in continuing bevacizumab therapy in patients with colorectal cancer following the development of nasal septal perforation, and advise a detailed discussion of risk with the patient.

\section{References}

[1] Ellis, L.M. (2006) Mechanisms of Action of Bevacizumab as a Component of Therapy for Metastatic Colorectal Cancer. Seminars in Oncology, 33, S1-S7.

[2] Whyte, S., Pandor, A. and Stevenson, M. (2012) Bevacizumab for Metastatic Colorectal Cancer: A NICE Single Te- 
chnology Appraisal. Pharmacoeconomics, 30, 1119-1132. http://dx.doi.org/10.2165/11597210-000000000-00000

[3] Mailliez, A., et al. (2010) Nasal Septum Perforation: A Side Effect of Bevacizumab Chemotherapy in Breast Cancer Patients. British Journal of Cancer, 103, 772-775. http://dx.doi.org/10.1038/sj.bjc.6605828

[4] Ramiscal, J.A. and Jatoi, A. (2011) Bevacizumab-Induced Nasal Septal Perforation: Incidence of Symptomatic, Confirmed Event(s) in Colorectal Cancer Patients. Acta Oncologica, 50, 578-581. http://dx.doi.org/10.3109/0284186X.2010.537692

[5] Mailliez, A., et al. (2010) Nasal Septum Perforations: A Side Effect of the Association of Bevacizumab and Taxanes in Patients with Breast Cancer? ASCO Meeting Abstracts, 28, 671.

[6] Hapani, S., Chu, D. and Wu, S. (2009) Risk of Gastrointestinal Perforation in Patients with Cancer Treated with Bevacizumab: A Meta-Analysis. The Lancet Oncology, 10, 559-568. http://dx.doi.org/10.1016/S1470-2045(09)70112-3

[7] Hompes, D. and Ruers, T. (2011) Review: Incidence and Clinical Significance of Bevacizumab-Related Non-Surgical and Surgical Serious Adverse Events in Metastatic Colorectal Cancer. European Journal of Surgical Oncology (EJSO), 37, 737-746. http://dx.doi.org/10.1016/j.ejso.2011.06.004

[8] Van Cutsem, E., et al. (2009) Safety and Efficacy of First-Line Bevacizumab with FOLFOX, XELOX, FOLFIRI and Fluoropyrimidines in Metastatic Colorectal Cancer: The BEAT Study. Annals of Oncology, 20, 1842-1847. http://dx.doi.org/10.1093/annonc/mdp233

[9] Kozloff, M., et al. (2009) Clinical Outcomes Associated with Bevacizumab-Containing Treatment of Metastatic Colorectal Cancer: The BRiTE Observational Cohort Study. The Oncologist, 14, 862-870. http://dx.doi.org/10.1634/theoncologist.2009-0071

[10] Poultsides, G.A., et al. (2009) Outcome of Primary Tumor in Patients with Synchronous Stage IV Colorectal Cancer Receiving Combination Chemotherapy without Surgery as Initial Treatment. Journal of Clinical Oncology, 27, 33793384. http://dx.doi.org/10.1200/JCO.2008.20.9817 